\title{
ZERO SUMS OF IDEMPOTENTS IN BANACH ALGEBRAS
}

\author{
H. Bart, T. Ehrhardt and B. Silbermann
}

The problem treated in this paper is the following. Let $p_{1}, \ldots, p_{k}$ be idempotents in a Banach algebra $B$, and assume $p_{1}+\ldots+p_{k}=0$. Does it follow that $p_{j}=0$, $j=1, \ldots, k$ ? For important classes of Banach algebras the answer turns out to be positive; in general, however, it is negative. A counterexample is given involving five nonzero bounded projections on infinite-dimensional separable Hilbert space. The number five is critical here: in Banach algebras nontrivial zero sums of four idempotents are impossible. In a purely algebraic context (no norm), the situation is different. There the critical number is four.

\section{INTRODUCTION}

In this paper we study the problem stated in the abstract. The problem is intriguing in its own right, but it came to the attention of the authors in their investigation of logaritmic residues in Banach algebras. This background, and more specifically the connection with [BES 1 , is briefly explained at the end of this introduction.

A full solution to the problem is not yet available (and might very well stay out of reach for some time to come). For a variety of important Banach algebras, however, positive results are obtained. Among these are the generalizations of commutative Banach algebras called polynomial-identity Banach algebras and certain Banach algebras that appear in the (numerical) work of S. Roch and B. Silbermann. An important instance of the latter category is the Banach subalgebra of $\mathcal{L}\left(L_{2}(\Pi)\right)$ generated by all compact operators on $L_{2}(\mathbb{T})$, all operators on $L_{2}(\mathbb{T})$ of multiplication by piece-wise continuous functions, and the operator $S$ of singular integration along $\mathbb{T}$; here $\mathbb{T}$ is the unit circle in the complex plane. This Banach algebra is not a polynomial-identity algebra.

In spite of the positive results indicated in the previous paragraph, the answer to the question under discussion is generally negative. This appears from a counterexample involving five nonzero bounded projections on infinite-dimensional separable Hilbert space. As an immediate consequence, one has that nontrivial zero sums of $k$ bounded projections on infinite dimensional Hilbert space exist for every 
$k$ larger than or equal to five.

The number five is critical here: Nontrivial zero sums of four idempotents in Banach algebras do not exist. This is especially remarkable because in a purely algebraic context, the critical number is not five, but four. The existence of rings with four nonzero idempotents adding up to zero was already established in [Ma]; see also [Be]. The approach in these papers is rather abstract. In the present paper we give an example involving a concrete algebra of linear operators.

As announced already, we conclude this introduction by briefly indicating the connection with $\left[\mathrm{BES}_{1}\right]$. In that paper, the authors introduce and investigate the concept of a logarithmic residue in a Banach algebra. A logarithmic residue is an element of the form

$$
\frac{1}{2 \pi i} \int_{\partial D} f^{\prime}(\lambda) f(\lambda)^{-1} d \lambda
$$

where the Banach algebra valued function $f$ and the Cauchy domain $D(\subset \mathbb{C})$ satisfy certain conditions (such as, for example, that $f$ is analytic in a neighbourhood of the closure of $D$ ). Each sum of idempotents in a Banach algebra is a logarithmic residue. So the problem discussed in the present paper is a special case of the broader issue of vanishing logarithmic residues. It is that issue that is addressed in $\left[B E S_{1}\right]$. The main results is $\left[B E S_{1}\right]$ are concerned with sufficient conditions under which the assumption that (1.1) vanishes implies that $f$ takes invertible values on $D$. Theorems of this type are vector valued versions of one of the basic results from complex function theory.

Acknowledgements: The foundation for this paper was laid when the first and last author were at the 20th Functional Analysis Seminar organized by V. Pták and P. Vrbobá in Liptovsky Jàn, Czechoslovakia, May-June 1989. The authors are grateful to T.J. Laffey for bringing to their attention the references [Be] and [Ma].

\section{BANACH ALGEBRAS WITHOUT NONTRIVIAL ZERO SUMS OF IDEMPOTENTS}

The problem discussed in this paper is the following: Let $B$ be a Banach algebra, and let $p_{1}, \ldots, p_{k}$ be idempotents in $B$ such that $p_{1}+\ldots+p_{k}=0$. Does it follow that $p_{j}=0, j=1, \ldots, k$ ? In this section we shall see that for important classes of Banach algebras, the answer is positive.

We begin with an elementary observation. For $X$ a Banach space, $\mathcal{L}(X)$ will denote the Banach algebra of all bounded linear operators on $X$. Note that the idempotents in $\mathcal{L}(X)$ are just the projection operators on $X$. 
PROPOSITION 2.1 Let $B=\mathcal{L}(X)$, where $X$ is a Banach space, and let $P_{1}, \ldots, P_{k}$ be idempotents in $B$ such that $P_{1}+\ldots+P_{k}=0$. Assume, in addition, that $P_{1}, \ldots, P_{k}$ are of finite rank. Then $P_{j}=0, j=1, \ldots k$.

PROOF Take traces and use that for a finite rank projection operator, trace and rank coincide.

For our next result, we need some notation and terminology. As before, the setting is a Banach algebra $B$ with unit element, here to be denoted by $e$. By $\mathbb{C}^{n \times n}$ we mean the Banach algebra of all complex $n \times n$ matrices. The unit element in $\mathbb{C}^{n \times n}$ is the $n \times n$ identity matrix $I_{n}$. Of course $\mathbb{C}^{n \times n}$ can be identified with $\mathcal{L}\left(\mathbb{C}^{n}\right)$.

A mapping $\varphi: B \rightarrow \mathbb{C}^{n \times n}$ is called an $n \times n$ matrix representation of $B$ if it is linear, continuous (bounded) and multiplicative. So a $1 \times 1$ matrix representation is just a multiplicative bounded linear functional on $B$. If $\varphi$ is an $n \times n$ matrix representation of $B$, then $\varphi(e)$ is an idempotent $n \times n$ matrix. Hence $\varphi(e)$ is invertible if and only if $\varphi(e)=I_{n}$, and in that case $\varphi\left(b^{-1}\right)=\varphi(b)^{-1}$ for each invertible $b \in B$. We call $\psi$ a matrix representation of $B$ if there exists $n$ such that $\psi$ is an $n \times n$ matrix representation for $B$. $A$ nonempty set $\mathcal{M}$ of matrix representations of $B$ is said to be a sufficient family of matrix representations for $B$ if for each $b \in B$ the following holds: $b$ is invertible if and only if $\operatorname{det} \psi(b) \neq 0$ for all $\psi \in M$.

This terminology is inspired by $N$. Krupnik $[K]$. In Section 29 of $[K]$, the characterization of Banach algebras possessing a sufficient family of matrix representations is identified as an open problem. For our purposes here it is of interest to note that the class of Banach algebras possessing a sufficient family of matrix representations is large. It contains all matrix algebras $\mathbb{C}^{n \times n}$ (take $M=\left\{I_{n}\right\}$ ) and all commutative Banach algebras with unit element (Gelfand Theory). More generally, each polynomial-identity Banach algebra possesses a sufficient family of matrix representations (cf. [K]). Recall that $B$ is called a polynomial-identity Banach algebra if there exist a positive integer $k$ and a polynomial $p\left(x_{1}, \ldots, x_{k}\right)$ in $k$ noncommuting variables $x_{1}, \ldots, x_{k}, \quad p \neq 0$, such that $p\left(b_{1}, \ldots, b_{k}\right)=0$ for arbitrary $b_{1}, \ldots, b_{k} \in B$. As an example of a polynomial-identity Banach algebra we mention $\mathbb{C}^{n \times n}$. In that case one can take for $p$ the so called standard polynomial involving $2 \mathrm{n}$ variables, i.e., the polynomial $\sum_{\sigma}(\operatorname{sgn} \sigma) x_{\sigma(1)} \ldots x_{\sigma(2 n)}$, where $\sigma$ runs through the symmetric group $S_{2 n}$ (cf.[AL]). For a proof of this result and more examples, see [K].

THEOREM 2.2 Suppose $B$ is a Banach algebra possessing a sufficient family of matrix representations. If $p_{1}, \ldots, p_{k}$ are idempotents in $B$ with $p_{1}+\ldots+p_{k}=0$, then $p_{j}=0, j=1, \ldots, k$.

This result can be viewed as a comment to Problem 12 in $[\mathrm{K}]$, Section 29; see however Remark 2.4 below. In light of [BES 1 , Theorem 4.1, Theorem 2.2 above is 
contained in $\left[\mathrm{BES}_{1}\right]$, Proposition 5.1. For the convenience of the reader, we present a simple direct proof.

PROOF Take $\psi \in \mathcal{M}$, where $\mathcal{M}$ is a sufficient family of matrix representations for $B$, and put $P_{j}=\psi\left(p_{j}\right)$. Then $P_{1}+\ldots+P_{k}=0$. Note that $P_{1}, \ldots, P_{k}$ are idempotents matrices (of the same size). Hence $P_{1}=\ldots=P_{k}=0$ by Proposition 2.1. Take $j \in\{1, \ldots, k\}$. Then $\psi\left(e-p_{j}\right)=I-P_{j}=I$, where $I$ is the identity matrix of the appropriate size. In particular $\psi\left(e-p_{j}\right)$ is invertible. Since $\psi \in \mathcal{M}$ was taken arbitrarily, it follows that $e-p_{j}$ is invertible. Clearly $\left(e-p_{j}\right) p_{j}=0$, and so $p_{j}=0$.

COROLLARY 2.3 Let $p_{1}, \ldots, p_{k}$ be commuting idempotents in a Banach algebra $B$, and assume $p_{1}+\ldots+p_{k}=0$. Then $p_{j}=0, j=1, \ldots, k$.

PROOF We may assume that $B$ is a commutative Banach algebra; otherwise replace $B$ by the Banach subalgebra of $B$ generated by $p_{1}, \ldots, p_{k}$. Since a commutative Banach algebra posesses a sufficient family of matrix representations (Gelfand Theory), Theorem 2.2 applies.

A more straighforward proof of Corollary 2.3 can be given along the lines suggested by the proof of Proposition 4.1 below. We leave the details to the reader.

REMARK 2.4 As was already noted, Theorem 2.2 is a special case of [BES1], Proposition 5.1. Specializing another result from [BES1], namely [BES 1 , Remark 5.3, we see that the statement of Theorem 2.2 is also true when $B$ is a Banach algebra of the type considered in $[\mathrm{Sm}]$, Theorem 2 (and [BES $]$, Theorem 4.2). Such Banach algebras appear in the (numerical) work of S. Roch and B. Silbermann (cf. [Sm]), and they need not possess a sufficient family of matrix representations. As an important example (cf.[K]), we mention the Banach subalgebra of $\mathcal{L}\left(L_{2}(\mathbb{T})\right.$ ) generated by all compact operators on $L_{2}(\mathbb{T})$, all operators on $L_{2}(\mathbb{T})$ of multiplication by piece-wise continuous functions and the operator $S$ of singular integration along $\mathbf{T}$; here $\mathbb{T}$ is the unit circle in the complex plane $\mathbb{C}$. This Banach algebra does not possess a sufficient family of matrix representations (see [BES1], Section 4). So it is not covered by Theorem 2.2; nevertheless the statement of the theorem holds true.

\section{A COUNTEREXAMPLE}

As we have seen in the previous section, the answer to the question discussed in this paper is positive for important classes of Banach algebras. In general, however, it is negative. Here is a (nonexotic) example.

EXAMPLE 3.1 Let $H$ be the Hilbert space obtained by taking the orthogonal direct sum of three copies of the sequence space $\ell_{2}: H=\ell_{2} \oplus \ell_{2} \oplus \ell_{2}$. The identity operator on $\ell_{2}$ will be denoted by $I$.

Let $a, b, c$ and $d$ be complex numbers such that $a c=3$ and $b d=\frac{-14}{5}$, and introduce 


$$
\begin{aligned}
& P_{1}=\frac{5}{6}\left(\begin{array}{rrr}
I & c I & d I \\
a I & a c I & a d I \\
b I & b c I & b d I
\end{array}\right): \ell_{2} \oplus \ell_{2} \oplus \ell_{2} \rightarrow \ell_{2} \oplus \ell_{2} \oplus \ell_{2} \\
& P_{2}=\frac{5}{6}\left(\begin{array}{rrr}
I & -c I & d I \\
-a I & a c I & -a d I \\
b I & -b c I & b d I
\end{array}\right): \ell_{2} \oplus \ell_{2} \oplus \ell_{2} \rightarrow \ell_{2} \oplus \ell_{2} \oplus \ell_{2} \\
& P_{3}=\frac{10}{3}\left(\begin{array}{rrr}
I & 0 & -\frac{1}{2} d I \\
0 & 0 & 0 \\
-\frac{1}{2} b I & 0 & \frac{1}{4} b d I
\end{array}\right): \ell_{2} \oplus \ell_{2} \oplus \ell_{2} \rightarrow \ell_{2} \oplus \ell_{2} \oplus \ell_{2} .
\end{aligned}
$$

Then $P_{1}, P_{2}, P_{3} \in \mathcal{L}(H)$ and

$$
P_{1}+P_{2}+P_{3}=\left(\begin{array}{rrr}
5 I & 0 & 0 \\
0 & 5 I & 0 \\
0 & 0 & -7 I
\end{array}\right)
$$

In order to introduce $P_{4}$ and $P_{5}$ we need to make some preparations. Define the bounded linear operators $T_{1}, T_{2}, S_{1}$ and $S_{2}$ on $\ell_{2}$ by stipulating that

$$
\begin{aligned}
& T_{1}\left(x_{0}, x_{1}, x_{2}, \ldots\right)=\left(x_{0}, 0, x_{1}, 0, x_{2}, 0, x_{3}, \ldots\right) \\
& T_{2}\left(x_{0}, x_{1}, x_{2}, \ldots\right)=\left(0, x_{0}, 0, x_{1}, 0, x_{2}, 0, \ldots\right) \\
& S_{1}\left(x_{0}, x_{1}, x_{2}, \ldots\right)=\left(x_{0}, x_{2}, x_{4}, x_{6}, \ldots\right) \\
& S_{2}\left(x_{0}, x_{1}, x_{2}, \ldots\right)=\left(x_{1}, x_{3}, x_{5}, x_{7}, \ldots\right) .
\end{aligned}
$$

Then the following identities are satisfied:

$$
T_{1} S_{1}+T_{2} S_{2}=I, \quad S_{1} T_{1}=S_{2} T_{2}=I, \quad S_{1} T_{2}=S_{2} T_{1}=0
$$

Now choose $\alpha$ and $\beta$ in $\mathbb{C}$ such that $\alpha \beta=\frac{-35}{4}$, and introduce

$$
\begin{aligned}
& P_{4}=\left(\begin{array}{rcc}
-\frac{5}{2} I & 0 & \beta S_{1} \\
0 & -\frac{5}{2} I & \beta S_{2} \\
\alpha T_{1} & \alpha T_{2} & \frac{7}{2} I
\end{array}\right): \ell_{2} \oplus \ell_{2} \oplus \ell_{2} \rightarrow \ell_{2} \oplus \ell_{2} \oplus \ell_{2} \\
& P_{5}=\left(\begin{array}{rcc}
-\frac{5}{2} I & 0 & -\beta S_{1} \\
0 & -\frac{5}{2} I & -\beta S_{2} \\
-\alpha T_{1} & -\alpha T_{2} & \frac{7}{2} I
\end{array}\right): \ell_{2} \oplus \ell_{2} \oplus \ell_{2} \rightarrow \ell_{2} \oplus \ell_{2} \oplus \ell_{2} .
\end{aligned}
$$


Then $P_{4}, P_{5} \in \mathcal{L}(H)$ and

$$
P_{4}+P_{5}=\left(\begin{array}{rrr}
-5 I & 0 & 0 \\
0 & -5 I & 0 \\
0 & 0 & 7 I
\end{array}\right)
$$

Combining (3.1) and (3.2), we see that $P_{1}+P_{2}+P_{3}+P_{4}+P_{5}=0$. Straightforward computations show that $P_{1}, P_{2}, P_{3}, P_{4}$ and $P_{5}$ are idempotents in $\mathcal{L}(H)$, i.e., bounded projections on $\mathrm{H}$.

COROLLARY 3.2 Let $H$ be an infinite-dimensional Hilbert space. Then, for $k \geq 5$, there exist $k$ different nonzero bounded projections $P_{1}, \ldots, P_{k}$ on $H$ such that $P_{1}+\ldots+P_{k}=0$.

PROOF If $Q_{1}, \ldots, Q_{m}$ are non-zero projections on $H$ that add up to zero, then at least one of these projections has infinite rank (cf. Proposition 2.1). Further, if $Q$ is a projection of infinite rank on $H$, then $Q$ can be written as $Q=Q^{\prime}+Q^{\prime \prime}$ where $Q^{\prime}$ is a projection on $H$ of rank 1 and $Q^{\prime \prime}$ is a projection on $H$ of infinite rank. So, it suffices to deal with the case $k=5$. We may also assume that $H$ is separable. But then $H$ is isomorphic to the othogonal direct sum of three copies of the sequence space $\ell_{2}$. This brings us back to Example 3.1 .

\section{ZERO SUMS OF FOUR IDEMPOTENTS}

The material contained in Section 3 induces the following question: What about zero sums of idempotents involving (at most) four terms? As we shall see, the answer to this question has some intriguing aspects. We begin by considering zero sums of three idempotents in a purely algebraic context.

PROPOSITION 4.1 Let $A$ be an algebra, and let $p, q$ and $r$ be idempotents in $A$. If $p+q+r=0$, then $p=q=r=0$.

The case where one actually has a zero sum of only two idempotents (for instance $r=0$ ) is extremely simple. Indeed, if $p$ and $q$ are idempotents and $p+q=0$, then $p=p^{2}=(-q)^{2}=q^{2}=q=-p$. For three idempotents the argument is slightly more involved.

PROOF Clearly $(p+q)^{2}=(-r)^{2}=r^{2}=r=-p-q \quad$ and $\quad(p+q)^{2}=p+q+p q+q p . \quad$ Hence $2 p+2 q+p q+q p=0$. From this we obtain

$$
\begin{aligned}
& 2 p+3 p q+p q p=p(2 p+2 q+p q+q p)=0 \\
& 2 p+3 q p+p q p=(2 p+2 q+p q+q p) p=0
\end{aligned}
$$


and these identities imply that $p q=q p$. So $p+q+p q=0$. Multiplying this identity from the left with $p$ and from the right with $q$, we get $p q=0$. It follows that $r=-(p+q)=0$. Analogously, $p=0$ and $q=0$.

In a purely algebraic context, an extension of Proposition 4.1 to zero sums involving four idempotents is not possible. In an abstract ring-theoretic framework, this has been established in $[\mathrm{Ma}]$; see also $[\mathrm{Be}]$. Here we present an example involving a concrete algebra of linear operators.

EXAMPLE 4.2 Let $X$ be the linear space of complex valued functions on the real line $\mathbb{R}$ endowed with the usual pointwise operations, and write $L(X)$ for the algebra of linear operators on $X$. Define $P, Q, R$ and $S$ in $L(X)$ by

$$
\begin{aligned}
& (P f)(x)=x[f(x)+f(1-x)] \\
& (Q f)(x)=x[f(x)-f(1-x)] \\
& (R f)(x)=x[-f(x)+f(-1-x)] \\
& (S f)(x)=x[-f(x)-f(-1-x)] .
\end{aligned}
$$

Then, obviously, $P+Q+R+S=0$. Straightforward computations show that $P^{2}=P, Q^{2}=Q, R^{2}=R$ and $S^{2}=S$.

We conclude by showing that Proposition 4.1 does allow for an extension to zero sums involving four idempotents when the context is that of normed algebras. This fact is remarkable in view of the previous example and Example 3.1.

THEOREM 4.3 Let $B$ be a Banach algebra and let $a, b, c$ and $d$ be idempotents in B. If $a+b+c+d=0$, then $a=b=c=d=0$.

It is not essential that $B$ has a unit element. In fact, one can always adjoin one. The assumption that $B$ is (topologically) complete also does not play a role in the proof. Crucial is that $B$ is a normed algebra (which, of course, can always be completed).

PROOF For idempotents $u$ and $v$, we have

$$
\begin{aligned}
& (u+v)^{2}+(u-v)^{2}=2(u+v) \\
& (u-v)(u+v)+(u+v)(u-v)=2(u-v) .
\end{aligned}
$$

These identities will be used frequently in what follows.

Put $p=(c-d)(a-b)$ and $q=(a-b)(c-d)$. Then

$$
\begin{aligned}
p(a+b) & =(c-d)(a-b)(a+b)=(c-d)[2(a-b)-(a+b)(a-b)] \\
& =2 p+(c-d)(c+d)(a-b)=2 p+[2(c-d)-(c+d)(c-d)](a-b) \\
& =4 p+(a+b) p .
\end{aligned}
$$


With induction, one can now easily prove that

$$
p^{m}(a+b)-(a+b) p^{m}=4 m p^{m}
$$

for all positive integers $m$ (and also for $m=0$ provided $p^{0}(a+b)$ and $(a+b) p^{0}$ are read as $a+b$ ). Taking norms, we get

$$
\left\|p^{m}\right\|[4 m-2\|a+b\|] \leqq 0 .
$$

It follows that $p^{m}=0$ for $m$ sufficiently large. In other words: $p$ is nilpotent. The same conclusion holds for $q$.

Let $y \in B, \lambda \in \mathbb{C}$ and assume

$$
(a+b) y=\lambda y .
$$

We shall prove that

$$
\begin{aligned}
& (a+b) q y=(\lambda+4) q y, \\
& p q y=\left(\lambda^{2}+6 \lambda+8\right)\left(\lambda^{2}+2 \lambda\right) y .
\end{aligned}
$$

This goes as follows. From (4.1) and $a+b+c+d=0$, we obtain $(c+d) y=-\lambda y$ and

$$
(c+d)(c-d) y=[2(c-d)-(c-d)(c+d)] y=(2+\lambda)(c-d) y .
$$

Hence $(a+b)(c-d) y=-(c+d)(c-d) y=(-2-\lambda)(c-d) y$. This gives, on the one hand

$$
\begin{aligned}
(a+b) q y & =(a+b)(a-b)(c-d) y=[2(a-b)-(a-b)(a+b)](c-d) y \\
& =2 q y-(-2-\lambda) q y=(\lambda+4) q y
\end{aligned}
$$

and, on the other,

$$
\begin{aligned}
\text { pqy } & =(c-d)(a-b)^{2}(c-d) y=(c-d)\left[2(a+b)-(a+b)^{2}\right](c-d) y \\
& =(c-d)\left[(-4-2 \lambda)(c-d) y-(-2-\lambda)^{2}(c-d) y\right]=-\left(8+6 \lambda+\lambda^{2}\right)(c-d)^{2} y \\
& =-\left(8+6 \lambda+\lambda^{2}\right)\left[2(c+d) y-(c+d)^{2} y\right]=-\left(8+6 \lambda+\lambda^{2}\right)\left[-2 \lambda y-\lambda^{2} y\right] \\
& =\left(8+6 \lambda+\lambda^{2}\right)\left(2 \lambda+\lambda^{2}\right) y .
\end{aligned}
$$

With this, (4.2) and (4.3) have been derived from (4.1).

Suppose, again, that $(4.1)$ is satisfied. Repeated application of (4.2) yields that 


$$
(a+b) q^{m} y=(\lambda+4 m) q^{m} y
$$

This identity holds for all non-negative integers $m$ (provided $q^{0} \mathrm{y}$ is read as $y$ ). Applying (4.3), with $y$ replaced by $q^{m} y$ and $\lambda$ by $\lambda+4 m$, now gives

$$
p q^{m+1} y=\left[\lambda^{2}+(8 m+6) \lambda+16 m^{2}+24 m+8\right] \cdot\left[\lambda^{2}+(8 m+2) \lambda+16 m^{2}+8 m\right] q^{m} y
$$

Now consider the equation $(a+b) x=2 x$. We shall prove that there is only one solution, namely $x=0$. Indeed, from (4.4), with $\lambda=2$ and $y=x$, one gets

$$
p q^{m+1} x=\left(256 m^{4}+1024 m^{3}+1472 m^{2}+896 m+192\right) q^{m} x .
$$

This identity holds for all non-negative integers $m$ (provided $q^{0} x$ is read as $x$ ). Since $q$ is nilpotent, a sufficiently high power of $q$ vanishes. But then it is clear from (4.5) that $x=0$.

Next we prove that, with $\varepsilon$ a nonzero scalar (later to be taken +1 or -1 ),

$$
(a+\varepsilon b) x=0
$$

implies $a x=b x=c x=d x=0$. The analogous result for $c$ and $d$ is of course also true. The argument is as follows. Clearly

$$
(a+b) a x=a^{2} x+b a x=a^{2} x-\varepsilon b^{2} x=a x-\varepsilon b x=2 a x .
$$

Hence $a x=0$ and $b x=-\varepsilon^{-1} a x=0$. From $c+d=-(a+b)$, it follows that $(c+d) x=0$, and so $c x=d x=0$ (same argument).

We are now ready to prove that $p x=0$ implies $a x=b x=c x=d x=0$. Indeed, $p x=0$ means $(c-d)(a-b) x=0$. Hence $a(a-b) x=b(a-b) x=0$, and so $(a-b)^{2} x=0$. But then

$$
(a+b)^{2} x=\left[2(a+b)-(a-b)^{2}\right] x=2(a+b) x,
$$

and so $(a+b) x=0$. This, in turn, implies that $a x=b x=c x=d x=0$. The same conclusion holds when $q x=0$.

Recall that $q$ is nilpotent. We claim that, in fact, $q=0$. For this, it is sufficient to establish that $q^{m}=0$ with $m \geqq 2$ implies $q^{m-1}=0$. This goes as follows. From $\quad 0=q^{m}=q \cdot q^{m-1}$, we get $\quad c q^{m-1}=d q^{m-1}=0 . \quad$ Hence $\quad(c-d) q^{m-1}=0$, i.e., $(c-d)(a-b)(c-d) q^{m-2}=0$. In other words $p(c-d) q^{m-2}=0$. Thus $a(c-d) q^{m-2}=0$ and $b(c-d) q^{m-2}=0$, and so $q^{m-1}=(a-b)(c-d) q^{m-2}=0$. 
Since $q=0$, we have $q a=q b=q c=q d=0$. But then $a^{2}=b^{2}=c^{2}=d^{2}=0$. This completes the proof because $a, b, c$ and $d$ are idempotents.

\section{REFERENCES}

[AL] Amitsur, S.A., Levitski, J.: Minimal identities for algebras, Proc. Amer. Math. Soc. 1 (1950), 449-463.

[Be] Bergman, G.M.: The diamond lemna for ring theory, Advances in Mathematics 29 (1978), 178-218.

[BES1] Bart, H., Ehrhardt, T., Silbermann, B.: Logarithmic residues in Banach algebras, Integral Equations and Operator Theory, to appear.

[K] Krupnik, N. Ya:: Banach Algebras with Symbol and Singular Integral Operators, Operator Theory: Advances and Applications, Vol. 26, Birkhäuser, Basel, 1987.

[Ma] Mauldon, J.G.: Nonorthogonal idempotents whose sum is idempotent, Amer. Math. Monthly 71 (1964), 963-973.

[Sm] Silbermann, B.: Symbol constructions and numerical analysis, in: Integral Equations and Inverse Problems (Lazarov, R. and Petkov, V. eds), Pitman Research Notes in Mathematics, Vol. 235, 1991, 241-252.

H. Bart

Econometrisch Instituut

Erasmus Universiteit Rotterdam

Postbus 1738

3000 DR ROTTERDAM

The Netherlands

T. Ehrhardt and B. Silbermann

TU Chemnitz-Zwickau

Fachbereich Mathematik

PSF 964

09009 CHEMNITZ

Germany

MCS: Primary 16A32, 16A38, 46H99

Secondary $45 \mathrm{E} 05$

Submitted: October 10, 1993 